\title{
Parasitosis intestinal en monos capuchinos cariblancos Cebus capucinus (Primates: Cebidae) de un área protegida en la provincia de Limón, noreste de Costa Rica
}

\author{
Misael Chinchilla ${ }^{1}$, Bernardo Urbani ${ }^{2}{ }^{3}$ Idalia Valerio $^{1} \&$ Juan Carlos Vanegas ${ }^{1}$ \\ 1. Laboratorio de Investigación, Universidad de Ciencias Médicas “Dr. Andrés Vesalio Guzmán” (UCIMED), San José, \\ Costa Rica. Fax: +506 2290-2685; chinchillacm@ucimed.com \\ 2. Department of Biology, School of Science, Aristotle University of Thessaloniki, Tesalónica, Grecia. \\ 3. Dirección actual: Centro de Antropología, Instituto Venezolano de Investigaciones Científicas. Caracas, Venezuela; \\ burbani@illinois.edu
}

Recibido 13-VII-2010. Corregido 07-VIII-2010. Aceptado 10-VIII-2010.

\begin{abstract}
Intestinal parasites in white-faced capuchin monkeys Cebus capucinus Primates: Cebidae) inhabiting a protected area in the Limón province of Northeastern Costa Rica. Deforestation of tropical forests is threatening monkey biodiversity and their health status, dependent of an ecologically undisturbed area. To asses this relationship, we analyzed parasite occurrence in their intestines. The study was conducted at the Estación Biológica La Suerte (EBLS), Limón, Costa Rica. The group of white-faced capuchin monkeys (Cebus capucinus) was observed between March and December of 2006. A total of 75 feces samples were obtained. Once a sample was collected, the eaten plant type was identified to family and species level, and feces were processed in the laboratory to determine parasite incidence. Results showed that Moraceae was the most represented family in the samples. Among parasites, Strongyloides spp. and Acanthocephala were the most common. Positive prevalence of parasites was found similar and independent of sex and age of capuchin individuals. Microsporids were mainly reported in feces associated with Piperaceae. A low presence of these parasites was found in samples associated with Myrtaceae, with possible anti-parasite active components. The occurrence of parasites was relatively high in EBLS, when compared to other regions in Costa Rica. The higher occurrence of parasites observed in capuchins at EBLS may be due to the fact that this rain forest is surrounded by areas affected by human activities. We suggest the promotion of research in neotropical primates parasitology, for a better comprehension of the parasite-host relationship, and in a long term, being able to understand the ecosystems where they coexist, and consequently, preserve the biodiversity of the whole region. Rev. Biol. Trop. 58 (4): 1335-1346. Epub 2010 December 01.
\end{abstract}

Key words: parasites, Strongyloides spp., Acanthocephala, Microsporidia, Moraceae, Myrtaceae, Cebus capucinus, Costa Rica.

El mono capuchino cariblanco (Cebus capucinus) habita en la mayoría de las selvas de Costa Rica y Centroamérica (Emmons 1997). Si bien esta especie de capuchinos ha sido blanco de múltiples estudios de campo en bosques secos (Fragaszy et al. 2004), hasta hace poco tiempo, sólo se había estudiado sistemáticamente y a largo plazo en dos bosques tropicales lluviosos de América Central: la isla de Barro Colorado en Panamá (Oppenheiner
1969) y la Reserva de Fauna Silvestre de Laguna Guimoreto en Honduras (Buckley 1983). Sólo recientemente fue estudiado en el bosque de la Estación Biológica La Suerte en el nororiente de Costa Rica (Urbani 2009, ver Materiales y Métodos). Es allí, en regiones costarricenses cubiertas por bosques lluviosos caribeños, donde se observan severas presiones antrópicas como la expansión ganadera y la creación de nuevas plantaciones -especialmente 
de banano y piña en el caso de la Provincia de Limón- (M. Chinchilla \& B. Urbani, observ. pers.). Es en aquellas regiones tropicales lluviosas, paradójicamente, donde se conoce poco sobre el rol de los capuchinos como importantes componentes de estos ecosistemas, a pesar de que se han reportado individuos de $C$. capucinus dispersando semillas intactas de 67 diferentes especies de árboles en Barro Colorado, contribuyendo de manera sustancial a la regeneración del bosque (Wehncke et al. 2003). Bajo los comprometidos escenarios arriba indicados se tiene como resultado la pérdida de biodiversidad, reducción de fuentes de agua y disminución de la calidad de los suelos tropicales (Chapman \& Peres 2001). La deforestación tiene efecto directo en las poblaciones humanas particularmente en las áreas rurales (Chapman \& Peres 2001). Por ello, en las regiones neo- y paleotropicales donde habitan primates, éstos tienen un rol preponderante en la regeneración de las selvas y son parte fundamental de este tipo de ecosistemas (Heymann 1993), de allí la necesidad de conocer en detalle los aspectos de su biología, como es el caso de sus parásitos.

En Costa Rica existen cuatro especies de primates no-humanos, a saber, el mono aullador de manto (Alouatta palliata Gray 1849), el mono araña o colorado (Ateles geoffroyi Kuhl 1820), el mono ardilla (Saimiri oerstedii Reinhardt 1872) y el mono capuchino cariblanca (Cebus capucinus Linnaeus 1758). En Costa Rica, la población de estos primates ha ido en continuo descenso, estando en riesgo de desaparición, con la potencial aparición de extinciones locales (Zaldívar et al. 2004). El caso de Costa Rica, conocido por ser un modelo latinoamericano en la preservación de su riqueza natural, aún enfrenta peligros en su biodiversidad, ya que si bien tiene un destacado sistema de parques nacionales, presenta una de las mayores tasas de deforestación en el continente (Terborgh 1992). Hoy en día se hacen grandes esfuerzos para contrarrestar tal deforestación (Obando 2009). Por su parte, aunque el ecoturismo es una empresa desarrollada en el país, la paradoja reside en que los niveles de deforestación para la industria maderera así como para crear espacios para la ganadería y agricultura persisten como graves problemas ecológicos. Esto afecta directamente a las poblaciones de primates, no sólo a escala de la desaparición de bosques para el uso normal de sus ámbitos hogareños y obtención de recursos alimenticios, sino también por el potencial aumento y riesgo de parasitismos en poblaciones de monos silvestres (Gillespi et al. 2008). Esto último debido a la convivencia más cercana con poblaciones humanas locales permanentes y grupos de turistas (Gilberth \& Dodds 1987).

En términos generales, existen pocos estudios de parasitosis intestinal en monos capuchinos (Phillips et al. 2004, Fragaszy et al. 2004). En Costa Rica, se ha realizado uno similar en $C$. capucinus cubriendo geográficamente tanto el lado Pacífico como Atlántico del país (Chinchilla et al. 2007). Este trabajo es idóneo como base para futuros análisis por su potencial comparativo. En el presente estudio, se informa sobre la presencia de microsporidios localizados en intestinos de primates neotropicales -con este estudio de caso en $C$. capucinus-, siendo éste un aspecto poco conocido. Consecuentemente, la presente investigación está dirigida a determinar la parasitosis intestinal y de microsporidiosis en C. capucinus en un bosque tropical lluvioso de Costa Rica. En términos generales, es un aporte al estudio de la interacción animal-habitat a nivel parasitológico-intestinal (Nunn \& Altizer 2006, Huffman \& Chapman 2009), esperando que permita utilizarse como referente para efectuar futuras evaluaciones para la protección efectiva de los primates costarricenses a partir de múltiples estudios de caso de parasitología animal de conservación (Gillespie et al. 2008)

\section{MATERIALES Y MÉTODOS}

Sitio de estudio: El estudio de campo se llevó a cabo en la reserva privada de la Estación Biológica La Suerte, EBLS (10²6' $\mathrm{N}-83^{\circ} 47^{\prime}$ W) a $100 \mathrm{~m}$. s.n.m. Esta zona protegida se localiza en el Distrito Rosa, Cantón de Pococí, Provincia de Limón, al noreste de 
Costa Rica. Esta reserva se ubica en la región demarcada como Área de Conservación Tortuguero (ACTO) (SINAE 2001), la cual presenta una vegetación clasificada como bosque muy húmedo tropical (Bolaños et al. 1993). En este lugar existen remanentes de la selva lluviosa de tierras bajas caribeñas/atlánticas. En el llamado "bosque pequeño" de la estación se identificaron predominantemente las especies de árboles Pentaclethra macroloba y Goethalsia meiantha, además de otras como Hieronyma alchorneoides y Calatola costarricenses. Dos tipos de vegetación, bosque primario y bosque secundario avanzado componen más de $60 \%$ del área de estudio. El bosque de la estación está rodeado por potreros de ganado vacuno y plantaciones diversas, así como una mezcla de pinos y árboles nativos, parches de palma de pejibaye y árboles pioneros.

En este sitio de estudio, la temperatura anual promedio es de $28.3^{\circ} \mathrm{C}$ y la precipitación anual promedio de $3214 \mathrm{~mm}$. Las especies de primates presentes en la EBLS son el mono capuchino cariblanco (Cebus capucinus), el aullador de manto (Alouatta palliata) y el araña centroamericano o colorado (Ateles geoffroyi). La tala de árboles, la remoción de flora, la pesca y la caza están absolutamente prohibidas.

Especies en estudio: Los monos capuchinos cariblancos (Cebus capucinus) se distribuyen desde Honduras hasta Colombia y es la única especie de este género en Centroamérica (Reid 1997). Entre los primates neotropicales, los capuchinos son particulares al exhibir un destacado conjunto de rasgos ecológicos y conductuales (Visalberghi \& McGrew 1997), además representan el único grupo neotropical en que se ha reportado la manipulación de los objetos como herramientas en el medio silvestre; también se caracterizan por una gran destreza manual y conductas exploratorias complejas, que les permiten manipular y forrajear en distintos nichos de los bosques donde habitan (Fragaszy et al. 2004).

El grupo de capuchinos estudiado se llama "La Yunai" (Urbani 2009), es un grupo habituado a la presencia de observadores que vive en el llamado "bosque pequeño" de la EBLS, así como en remanentes de bosque de algunas propiedades aledañas de la población La Primavera, Cantón de Pococí. Este grupo estaba compuesto para finales del período de estudio (Diciembre 2006) de cuatro hembras adultas, una hembra sub-adulta, dos machos adultos, un macho sub-adulto, un macho juvenil y dos juveniles de sexo no determinado. Ningún animal fue tocado o manipulado durante el período de estudio.

Colección de muestras: El grupo de capuchinos cariblancos (Cebus capucinus) se observó durante 161 días, entre marzo y diciembre de 2006. Se registraron los datos conductuales usando la técnica de muestreo focal en los animales (Martin \& Bateson 2000), con intervalos de dos minutos. El mono focal se siguió continuamente a lo largo del día desde el crepúsculo hasta el atardecer. Los sitios donde los monos duermen se localizaron cada tarde, para retomar el muestreo conductual al día siguiente. Se recolectó información detallada sobre ubicación, hora de observación, edad-sexo del individuo, actividad en curso, dieta y árbol en uso, entre otras (Urbani 2009).

Un total de 75 muestras fecales fueron colectadas de animales que se encontraban bajo observación. Las muestras fueron tomadas de la cobertura de suelo del bosque, después de un breve intervalo de tiempo entre el momento de haber sido defecadas y su recolección. Las mismas se colocaron en alcohol a $70^{\circ}$. Las muestras de heces fueron revisadas cuidadosamente in situ, -y luego ex situ, en el laboratorio-, para determinar el tipo de semillas y/o otras partes (ej. endocarpio en el caso de Inga o elementos del núcleo de las semillas de Dipterix) de las frutas ingeridas y consecuentemente identificar las familias y especies de plantas asociadas con las heces. Para los datos recopilados se establecieron las relaciones correspondientes entre los parásitos encontrados, el sexo y la madurez de los individuos, así como con el tipo de plantas consumidos por ellos.

Procesamiento de muestras en el laboratorio: Las heces fueron conservadas en alcohol 
de $70^{\circ}$ para su transporte. En el laboratorio se realizaron los siguientes estudios: (a) análisis en directo, para lo cual se homogenizaron las muestras y se colocaron en solución salina al $85 \%$ y en lugol D'Antoni para el estudio de los parásitos intestinales comunes. Con el objeto de determinar la presencia de Cryptosporidium spp., Isospora spp, Cyclospora spp.y microsporidios, (b) tinciones de Koster modificado a fin de detectar Cryptosporidium spp. (Kageruka et al. 1984), (c) tinciones de Ziehl Nielsen para observar la presencia de Isospora spp. y Cyclospora spp. (Murray et al. 1995) y (d) tinciones de Weber para hallar esporas de microsporidios (Weber et al. 1992). Igualmente, en cada una de estas preparaciones se estudiaron 50 campos utilizando un lente 100x (Microscopio Olympus ${ }^{\mathrm{TM}}$ BX41).

Analisis estadísticos: Los datos fueron organizados en tablas de frecuencia. Como estas violan normalmente presupuestos de azar y a su vez tienden a no estar ubicadas en forma natural se utilizaron pruebas estadísticas no paramétricas (Sokal \& Rohlf 1995). En este sentido, los valores numéricos fueron sometidos a la prueba $G_{a d j}$, mientras que los valores porcentuales a la prueba $\chi^{2}$ (Fowler et al. 1998). El programa estadístico PopTools (acceso libre: http://www.cse.csiro.au/poptools) instalado en Excel $^{\mathrm{TM}}$ fue seleccionado para realizar los análisis estadísticos.

\section{RESULTADOS}

Una exhaustiva revisión de las heces permitió determinar las familias y especies de plantas ingeridas por los capuchinos. Las familias Moraceae y Fabaceae fueron las predominantes en las muestras $\left(G_{a d j}=84.31\right.$, d.f. $\left.=7, \mathrm{p}<0.01\right)$. Al tener estas dos familias la mayor representación en las muestras, era predecible que diferentes especies de Ficus e Inga, respectivamente, fueran las más comunes $\left(G_{a d j}=97.95\right.$, d.f. $=15$, $\mathrm{p}<0.01)$. Otras familias, al menos cinco, también se registraron en las muestras. El Cuadro

CUADRO 1

Familias y especies de plantas halladas en las heces de Cebus capucinus

TABLE 1

Plant families and species found in feces of Cebus capucinus

\begin{tabular}{|c|c|c|c|c|c|}
\hline Familia & Casos $(\mathrm{N})$ & Casos Porcentaje & Especies & Casos $(\mathrm{N})$ & Casos Porcentaje \\
\hline \multirow[t]{3}{*}{ Moraceae } & 35 & 42.7 & Ficus americana & 30 & 36.6 \\
\hline & & & Ficus spp. & 4 & 4.9 \\
\hline & & & Brosimum alicastrum & 1 & 1.2 \\
\hline \multirow[t]{6}{*}{ Fabaceae } & 20 & 24.4 & Dipterix panamensis & 9 & 11.0 \\
\hline & & & Inga spectabilis & 5 & 6.1 \\
\hline & & & Inga marginata & 3 & 3.7 \\
\hline & & & Inga oerstediana & 1 & 1.2 \\
\hline & & & Inga ruziana & 1 & 1.2 \\
\hline & & & Inga spp. & 1 & 1.2 \\
\hline \multirow[t]{2}{*}{ Melastomataceae } & 12 & 14.6 & Miconia affinis & 11 & 13.4 \\
\hline & & & Conostegia xalapensis & 1 & 1.2 \\
\hline Myrtaceae & 6 & 7.3 & Psidium guayaba & 6 & 7.3 \\
\hline Piperaceae & 3 & 3.7 & Piper sancti-felicis & 3 & 3.7 \\
\hline Annonaceae & 1 & 1.2 & Guatteria inuncta & 1 & 1.2 \\
\hline Passifloraceae & 1 & 1.2 & Passiflora ambigua & 1 & 1.2 \\
\hline Desconocida & 4 & 4.9 & gen. sp. desconocido & 4 & 4.9 \\
\hline Total & 82 & & & 82 & \\
\hline
\end{tabular}


1 resume la información de familias y especies de plantas ingeridas y halladas en las heces de los capuchinos.

La distribución positiva de parásitos en las muestras fue hallada de forma relativamente similar para ambos sexos (no estadísticamente significativo para $\bigcirc$ : $G_{a d j}=3.95$, d.f. $=1$, $\mathrm{p}>0.05$, aunque significativa en $\mathrm{O}^{7}: G_{a d j}=6.15$, d.f.=1, p<0.05), así como para aquellos cuyo sexo no se pudo determinar $\left(G_{a d j}=5.65\right.$, d.f. $=1$, p <0.05). El Cuadro 2 muestra la distribución de la presencia de parásitos por sexo. De igual forma, se encontró que independientemente de la edad, la positividad en torno a la presencia de parásitos en las muestras es predominante en ambos, jóvenes $\left(G_{a d j}=4.94\right.$, d.f. $\left.=1, \mathrm{p}<0.05\right)$ y adultos $\left(G_{a d j}=8.50\right.$, d.f. $\left.=1, \mathrm{p}<0.01\right)$. El Cuadro 3 presenta la información sobre la proporción de parasitismo de acuerdo con la madurez fisiológica o edad.
En cuanto a los parásitos intestinales encontrados en las muestras de heces de $C$. capucinos, se observó una mayor existencia de Strongyloides spp. (Fig. 1), superando y duplicando su presencia positiva con respecto a los acantocéfalos (Fig. 2), que se ubican de segundo en la lista de prevalencia parasitaria $\left(G_{a d}=60.00\right.$, d.f. $\left.=7, \mathrm{p}<0.01\right)$. Por otra parte, destaca, no por sus valores cuantitativos, sino por su particularidad, la existencia de infecciones múltiples parasitarias. El Cuadro 4 indica los parásitos hallados en $C$. capucinus.

El Cuadro 5 muestra información sobre la presencia de parásitos en relación con la familia de planta hallada en la muestra de heces. En este sentido, por ejemplo, Strongyloides spp. es predominante, aunque no estadísticamente significativo, en muestras con evidencia de consumo de la familia Moraceae $\left(G_{a d j}=5.55\right.$, d.f. $=3, \mathrm{p}>0.05$ ) o en la familia Melastomataceae

CUADRO 2

Distribución parasitaria por sexo en C. capucinus

TABLE 2

Parasitological distribution by sex in $\mathrm{C}$. capucinus

\begin{tabular}{lccccc}
\multicolumn{1}{r}{ Sexo } & Muestra $(\mathrm{N}) *$ & Positivo $(\mathrm{N})$ & Positivo (\%) & Negativo (N) & Negativo (\%) \\
Hembras & 25 & 16 & 64.0 & 9 & 36.0 \\
Machos & 24 & 18 & 75.0 & 6 & 25.0 \\
No definidos & 26 & 19 & 73.1 & 7 & 26.9 \\
Total & 75 & 53 & - & 22 & -
\end{tabular}

*Porcentaje calculados del total por cada sexo.

CUADRO 3

Proporción parasitaria a nivel de madurez fisiológica en $\mathrm{C}$. capucinus

TABLE 3

Parasitism proportion at an age level found in $\mathrm{C}$. capucinus

\begin{tabular}{|c|c|c|c|c|c|}
\hline Edad & Muestra $(\mathrm{N})^{*}$ & Positivo (N) & Positivo (\%) & Negativo (N) & Negativo (\%) \\
\hline Jóvenes** & 40 & 27 & 67.5 & 13 & 32.5 \\
\hline Adultos & 35 & 26 & 74.3 & 9 & 25.7 \\
\hline Total & 75 & 53 & - & 22 & - \\
\hline
\end{tabular}

*Porcentaje calculado del total en cada estado de madurez fisiológica o edad.

** Se refiere a individuos juveniles y sub-adultos. 


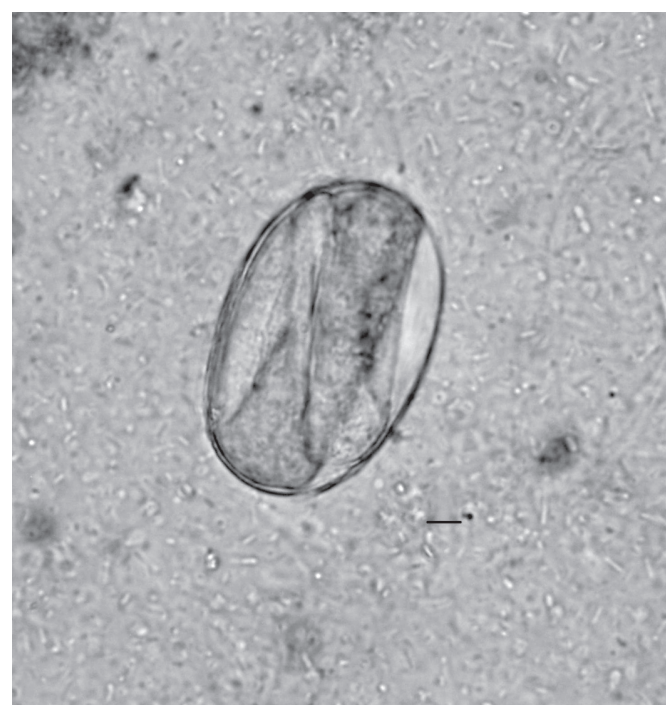

Fig. 1. Huevecillo de Strongyloides spp., parásito prevalente en muestras de heces de Cebus capucinus: (barra de escala: $5 \mu \mathrm{m})$.

Fig. 1. Egg of Strongyloides spp., prevalent parasite in feces of Cebus capucinus: (scale bar: $5 \mu \mathrm{m})$.

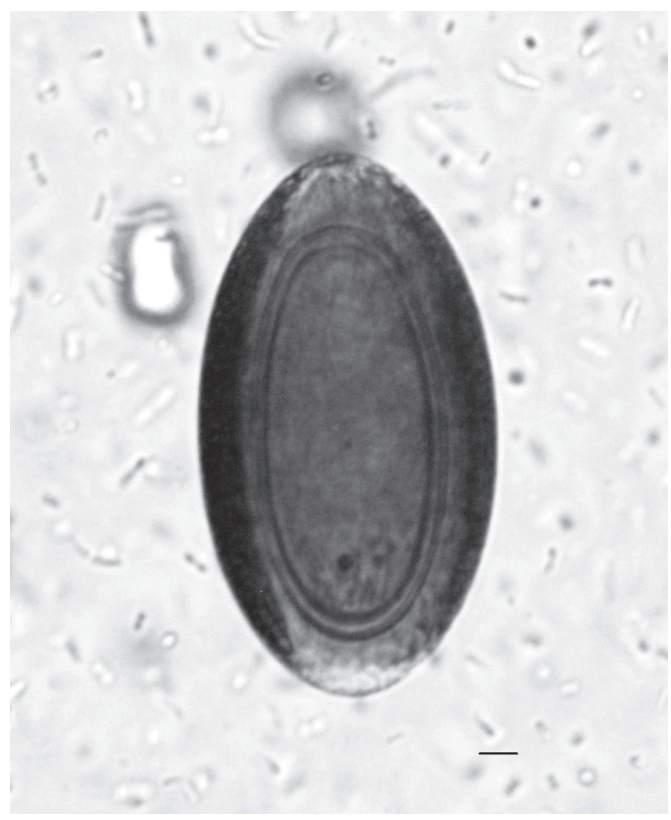

Fig. 2. Huevecillo de acantocéfalo, parásito comúnmente hallado en muestras de heces de $C$. capucinus: (barra de escala: $12 \mu \mathrm{m})$.

Fig. 2. Egg of Acantocephala, parasite commonly found in feces of C. capucinus: (scale bar: $12 \mu \mathrm{m})$.

\section{CUADRO 4}

Parásitos intestinales hallados en $\mathrm{C}$. capucinus

TABLE 4

Intestinal parasites found in $\mathrm{C}$. capucinus

\begin{tabular}{|c|c|c|}
\hline Parásito & Positivo (N) & Positivo (\%) \\
\hline Strongyloides spp. & 27 & 36.0 \\
\hline Acantocéfalos & 12 & 16.0 \\
\hline Uncinarias & 9 & 10.6 \\
\hline Uncinarias + Strongyloides spp. & 2 & 2.7 \\
\hline Strongyloides spp. + Acantocefalos & 2 & 2.7 \\
\hline Uncinarias + Strongyloides spp. + Acantocéfalos & 1 & 1.3 \\
\hline Uncinarias + Strongyloides spp. + Ascárido & 1 & 1.3 \\
\hline Total* & 54 & 72.0 \\
\hline
\end{tabular}

* Negativo $(\mathrm{N})=21 ;$ Negativo $(\%)=28.0$. 
CUADRO 5

Parásitos intestinales encontrados frecuentemente en C. capucinus con relación a las familias de plantas ingeridas

TABLE 5

Intestinal parasites frequently found in $\mathrm{C}$. capucinus in relation to the ingested plant families

$\begin{array}{lccccc}\text { Familia de planta } & \begin{array}{c}\text { Muestra } \\ (\mathrm{N})^{*}\end{array} & \begin{array}{c}\text { Strongyloides spp.: } \\ \mathrm{N}(\%)\end{array} & \begin{array}{c}\text { Acantocéfalos: } \\ \mathrm{N}(\%)\end{array} & \begin{array}{c}\text { Uncinarias: } \\ \mathrm{N}(\%)\end{array} & \begin{array}{c}\text { Negativo: } \\ \mathrm{N}(\%)\end{array} \\ \text { Moraceae } & 35 & 15(42.9) & 8(22.8) & 6(17.1) & 6(17.1) \\ \text { Fabaceae } & 20 & 5(25.0) & 2(10.0) & 5(25.0) & 8(40.0) \\ \text { Melastomataceae } & 12 & 7(58.3) & 3(25.0) & - & 2(16.7) \\ \text { Myrtaceae } & 6 & 2(33.3) & 1(16.7) & - & 3(50.0) \\ \text { Otros** } & 9 & 2(22.2) & 2(22.2) & - & 5(55.5) \\ \text { Total } & 81 & 32(39.5) & 15(18.5) & 11(13.6) & 23(28.4)\end{array}$

*Porcentaje calculados del total en cada familia de plantas.

**Annonaceae $(\mathrm{N}=1)$, Passifloraceae $(\mathrm{N}=1)$, Piperaceae $(\mathrm{N}=3)$, familia desconocida $(\mathrm{N}=4)$.

$\left(G_{a d j}=5.05\right.$, d.f. $\left.=3, \mathrm{p}>0.05\right)$. Destaca a su vez, la ausencia de uncinarias en las muestras asociadas a Myrtaceae. Con respecto a las fabáceas, la distribución de Strongyloides spp., acantocéfalos y uncinarias es relativamente uniforme en muestras con existencia de esta familia de plantas $\left(G_{a d j}=3.70\right.$, d.f. $=3$, p $\left.>0.05\right)$.

La presencia de microsporidios (Fig. 3) fue mayor, estadísticamente muy significativo, en muestras asociadas con la familia Piperaceae $\left(G_{a d j}=42.33\right.$, d.f. $\left.=1, \mathrm{p}<0.01\right)$, siendo negativo en el resto de las familias de plantas (Cuadro
6). Finalmente, cuando se comparan los porcentajes de parasitismo de los primates de la EBLS con las frecuencias encontradas en el resto de Costa Rica, no se encontró diferencia significativa en hembras (EBLS $=64.0 \%$ vs resto del país [RP]: $50 \% \cdot \chi^{2}=1.72$, d.f. $=1$, $\mathrm{p}>0.05)$. Sin embargo, cuando se compararon las muestras de machos (EBLS $=75.0 \%$ vs. $\mathrm{RP}=48.7 \% \cdot \chi^{2}=5.59$, d.f. $\left.=1, \mathrm{p}<0.05\right)$ y los totales de ambos sexos (EBLS=70.7\% vs. $\mathrm{RP}=19.1$. $\chi^{2}=29.65$, d.f. $=1, \mathrm{p}<0.01$ ), se hallaron diferencias hacia mayor parasitismo en las muestras de

\section{CUADRO 6}

Microsporidios hallados en C. capucinus con relación a las familias de plantas ingeridas

TABLE 6

Microporidia found in $\mathrm{C}$. capucinus in relation to the ingested plant families

\begin{tabular}{lccccc}
\multicolumn{1}{c}{ Familia de planta* } & Muestra $(\mathrm{N}) * *$ & Positivo $(\mathrm{N})$ & Positivo $(\%)$ & Negativo (N) & Negativo $(\%)$ \\
Moraceae & 35 & 8 & 25.0 & 24 & 75.0 \\
Fabaceae & 20 & 1 & 5.0 & 19 & 95.0 \\
Melastomataceae & 12 & 2 & 16.7 & 10 & 83.3 \\
Piperaceae & 3 & 2 & 66.7 & 1 & 33.3 \\
Otros*** & 6 & 4 & 66.7 & 2 & 33.3 \\
Total & 72 & 16 & 22.2 & 56 & 77.8
\end{tabular}

* Ninguno de los cinco individuos que ingirieron plantas de la familia Myrtaceae presentaron microsporidios.

**Porcentaje calculados del total en cada familia de plantas.

***Anonaceae $(\mathrm{N}=1)$, Passifloraceae $(\mathrm{N}=1)$, familia desconocida $(\mathrm{N}=4)$. 
la EBLS. Sin embargo estas diferencias deben ser contextualizadas para entender el patrón encontrado.

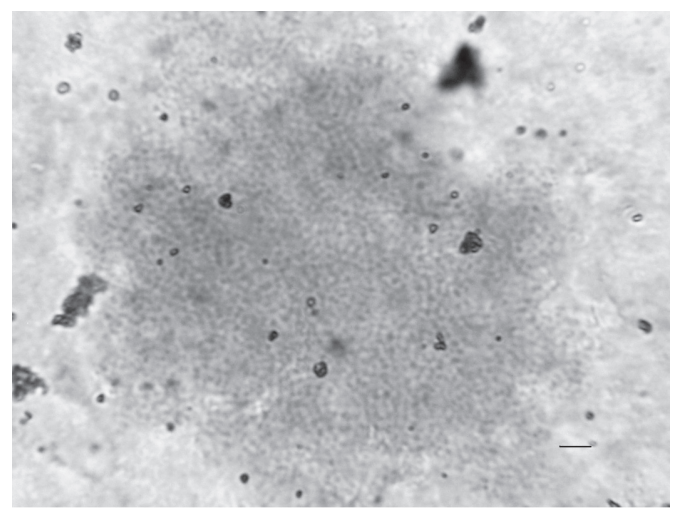

Fig. 3. Grupo de esporas de microsporidios hallado en muestras de heces de C. capucinus (barra de escala: $26 \mu \mathrm{m})$.

Fig. 3. Spores of Microsporidia found in feces of $C$.

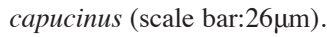

\section{DISCUSIÓN}

Se han publicado múltiples estudios sobre parasitología intestinal en primates silvestres del Neotrópico (Hugot et al. 1995, Stuart et al. 1998, Stoner et al. 2005). Sin embargo, para los primates de Costa Rica, si bien se han realizado varios estudios, pocos reúnen un amplio esfuerzo de muestreo, tanto en cantidad de ejemplares como en colectas de heces para su estudio parasitológico. El mono aullador de manto (Alouatta palliata) ha sido la especie más estudiada en Centroamérica y Costa Rica (véase Chinchilla et al. 2005, Villalobos et al. 2004). En el caso de los capuchinos y en particular Cebus capucinus en Costa Rica, los trabajos son aún más escasos (Troyo et al. 2004, Chinchilla et al. 2007). Sin embargo este último (Chinchilla et al. 2007) se realizó cubriendo prácticamente todo el país, colectándose muestras de 53 individuos.

Los parásitos encontrados en la EBLS, como Strongyloides spp., uncinarias y acantocéfalos, son similares a los reportados para esta especie en otras localidades de Costa Rica. Por otra parte, el índice de parasitosis general y por sexo fue alto en la EBLS si se le compara con lo reportado en otros sitios del país (Chinchilla et al. 2007). Sin embargo, estos valores son los esperados si consideramos que en ese estudio de un total de 53 muestras, $88.7 \%$ (47/53) corresponden a heces colectadas en localidades ubicadas en bosques secos y/o muy estacionales del lado Pacífico y $11.3 \%$ (6/53) corresponden a dos localidades cercanas a Sarapiquí y selvas muy húmedas del Atlántico costarricense. Estando las muestras balanceadas hacia los casos de poblaciones de capuchinos cariblancos de bosques más secos, se puede entender que las muestras de la EBLS presenten una diferencia notable con la información presentada en Chinchilla et al. (2007). En este sentido, es conocido que primates que habitan bosques muy húmedos, como la EBLS, tienden a poseer mayor frecuencia parasitaria que aquellas poblaciones de otros tipos de hábitats más secos (Hausfater \& Meade 1982, Stuart \& Strier 1995, Gillespi 2006, Huffman \& Chapman 2009), Por ejemplo, Stuart et al. (1998) halló que poblaciones de monos aulladores (Alouatta spp.) de localidades más húmedas, dentro de su distribución biogeográfica, presentaban mayor prevalencia parasitaria que aquellos encontrados en Alouatta palliata de un sitio del Pacífico costarricense. La segunda razón para tal diferenciación de valores posiblemente pueda deberse al hecho que "el bosque pequeño" de la EBLS, donde habitan los capuchinos estudiados, está rodeada de potreros y cultivos. Es sabido que la deforestación y presencia de cambios antrópicos pueden impactar de forma significativa la distribución y expresión de parásitos en primates silvestres (Gillespie \& Chapman 2006, Gillespie 2006).

Considerando las familias de plantas ingeridas, se nota una mayor presencia parasitaria en muestras de heces asociadas a las Moraceae y Melastomataceae. Por el contrario, la infección es menor porcentualmente en aquellas muestras de heces asociadas con restos de frutos de las familias Fabaceae y Myrtaceae. Esto 
explica, por ejemplo, en el caso de la familia Moraceae, que especies del género Ficus sean una de las más consumidas por los capuchinos en la EBLS (Urbani 2009). Sin embargo, es particularmente interesante notar que se han hallado componentes activos antiparasitarios específicamente en el género Psidium (Myrtaceae) (Costa-Brandelli et al. 2009), otras de las familias consumidas por los capuchinos de la EBLS.

Si bien el número de muestras positivas por microsporidios fue relativamente bajo, la tendencia en cuanto a su relación con la ingesta de plantas fue similar. Por eso en la familia Fabaceae solo 5\% fueron positivas. Nuevamente, como se indicó arriba, ningún individuo que ingirió plantas de la familia Myrtaceae resultó positivo. No existen estudios de plantas activas contra microsporidios, lo cual resulta interesante cuando se nota su ausencia al estar la familia Myrtaceae asociada con esas muestras de heces. El hecho que los capuchinos en general (Fragaszy et al. 2004) y los capuchinos cariblancos de la EBLS en particular (Urbani 2009), forrajean constantemente por insectos, puede explicar la presencia de microsporidios, ya que son frecuentes en estos invertebrados. Por su parte, no parece extrañar la ausencia de Cyclospora spp. y Cryptosporidium spp. en Cebus pues los informes en primates mencionan la presencia de estos organismos sólo en Ateles spp. (Stoner et al. 2005). Nosotros hemos encontrado también estos organismos en A. palliata (M. Chinchilla, datos inéditos). Otro aspecto que podría incidir en la ausencia de estos coccidios es la presencia de compuestos activos, tales como alcaloides, flavonoides y quinonas en algunas familias de plantas (Myrtaceae, Moraceae, Annonaceae) que fueron consumidas por los capuchinos de la EBLS y que se ha reportado actúan contra organismos del grupo de los Apicomplexa (Chinchilla et al. 2007). Por otro lado, para el caso de los seres humanos, la infección con Cryptosporidium spp. se ha encontrado más comúnmente por ingesta de agua y hortalizas contaminadas (Ho \& López 2002, Ortega et al. 1997, Luna et al. 2003, Calvo et al. 2004), mientras que
Cyclospora spp. se vincula más con plantas de la familia de las rosáceas (ej. frambuesas) o similares (Ho \& López 2002), lo cual no parece ser el caso de estos capuchinos.

En conclusión, este estudio es un aporte al conocimiento de los parásitos en primates costarricenses del género Cebus. Contribuir con este tipo de información, permitirá, eventualmente, discernir sobre situaciones de potenciales casos de zoonosis, lo cual podría representar inconvenientes no sólo para la salud pública sino también tener efectos deletéreos sobre la biodiversidad. Por ejemplo, existen reportes de parásitos normalmente hospedados en humanos transmitidos a primates y viceversa (Verweij et al. 2003), tal como se ha reportado en Costa Rica para otras especies de monos (Chinchilla et al. 2005). El crecimiento de las poblaciones humanas, sin duda, presiona peligrosamente a las selvas donde habitan los primates, posibilitando el potencial intercambio de patógenos, lo cual pudiera ser una amenaza para esas poblaciones y a su vez puede influir en las disminuciones -o extinciones- locales de poblaciones de primates no-humanos (Gillespie et al. 2008). Este tipo de investigaciones deben continuarse con el fin de lograr, cualitativa y cuantitativamente, mejores bases de datos sobre las interrelaciones parásitos-hospederos que a largo plazo contribuyan a comprender y por lo tanto preservar la biodiversidad de las selvas tropicales.

\section{AGRADECIMIENTOS}

Este estudio parasitológico fue financiado, en parte, por la Junta Administrativa y Rectoría a través de la Vicerrectoría Académica de la Universidad de Ciencias Médicas "Dr. Andrés Vesalio Guzmán” (UCIMED). Se agradece a Paul A. Garber por su cooperación durante el desarrollo del estudio de campo. Igualmente, a la familia Molina, por su apoyo durante el estudio en la EBLS, especialmente a Renee y Álvaro Molina; y a la familia Mesen-Rubí, en particular a Jonathan Mesen-Rubí por su participación en el campo. Gracias a Ricardo Vázquez (Museo Nacional de Costa Rica) por su 
cooperación y a Reinaldo Aguilar (INBIO) por su colaboración en la identificación de plantas. El estudio de campo doctoral de B. Urbani fue apoyado por subvenciones de la National Science Foundation, Graduate College-UIUC, American Society of Primatologists, Idea Wild Foundation, Departamento de AntropologíaUIUC y la American Philosophical Society. Los estudios doctorales de B.U. fueron sufragados por el Fulbright-OAS Ecology Initiative Program (LASPAU), Graduate College-UIUC y el Departamento de Antropología-UIUC, mientras que la estancia post-doctoral por la Greek State Scholarship Foundation (IKY) y la Aristotle University of Thessaloniki/Grecia.

\section{RESUMEN}

La deforestación de bosques tropicales está amenazando la biodiversidad de las especies de primates y su estado de salud, dependiente de un lugar ecológicamente equilibrado. Con el fin de evaluar esta relación, se analizó la presencia de parásitos en los intestinos de esta especie. El estudio se realizó en la Estación Biológica La Suerte (EBLS), Limón, Costa Rica. El grupo de monos capuchinos cariblancos (Cebus capucinus) fue observado entre marzo y diciembre de 2006. Se recolectaron 75 muestras de heces a las que se les identificó las plantas ingeridas, y se procesaron en el laboratorio para evaluar la prevalencia de parásitos. Moraceae fue la familia de plantas más reportada. Strongyloides spp. y acantocéfalos fueron los más comunes. La distribución positiva de las mismas fue similar independientemente del sexo y la edad. Microsporidios se reportaron mayoritariamente en heces asociadas con la familia Piperaceae. Fue encontrada una baja existencia de éstos parásitos en muestras asociadas con Myrtaceae en cuyo género Psidium, se han reportado compuestos activos antiparasitarios. La aparición de parásitos fue relativamente mayor en la EBLS, comparado con muestras de otras regiones más secas de Costa Rica. Por lo tanto, esa expresión parasitaria puede deberse al hecho de ser la EBLS una selva lluviosa, además de estar rodeada por zonas con actividades antrópicas. Se sugiere incrementar las investigaciones de parasitología de campo en primates neotropicales con el fin de entender plenamente las relaciones parásitos-hospederos, para en un largo plazo poder comprender los ecosistemas donde conviven, y en consecuencia, preservar la diversidad biológica.

Palabras clave: parásitos, Strongyloides spp., acantocéfalos, microsporidios, Moraceae, Myrtaceae, Cebus capucinus, Costa Rica.

\section{REFERENCIAS}

Bolaños, R., V. Watson \& J. Tosi. 1993. Mapa Ecológico de Costa Rica según el Sistema de Clasificación de Zonas de Vida del Mundo. Centro Científico Tropical-ICE, San José, Costa Rica. Escala 1:200 000.

Buckley, J.S. 1983. The feeding behavior, social behavior, and ecology of the white-faced monkey, Cebus capucinus at Trujillo, northern Honduras, Central America. Tesis de dotorado., Universidad de Texas, Austin, EEUU.

Calvo, M., M. Carazo, M.L. Arias, C. Chaves, R. Monge \& M. Chinchilla. 2004. Prevalencia de Cyclospora sp., Cryptosporidium sp., microsporidios y determinación de coliformes fecales en frutas y vegetales frescos de consumo crudo en Costa Rica. Arch. lat. Nut. ALAN 54: 428-432.

Chapman, C.A. \& C.A. Peres. 2001. Primate conservation in the New Millenium: The role of scientists. Evol. Anthropol. 10: 16-33.

Chinchilla, M., O. Guerrero, G.A. Gutiérrez-Espeleta, R. Sánchez Porras \& B. Rodríguez- Ortiz. 2005. Parásitos intestinales en monos congo Alouatta palliata (Primates: Cebidae) de Costa Rica. Rev. Biol. Trop. 53: 437-445.

Chinchilla, M., O. Guerrero, G.A. Gutiérrez-Espeleta, R. Sánchez \& I. Valerio Campos. 2007. Parásitos en monos carablanca Cebus capucinus (Primates: Cebidae) de Costa Rica. Parasitol. Latinoam. 62: 170-176.

Costa Brandelli, C.L., R. Brandt Giordani, G.A. De Carli \& T. Tasca. 2009. Indigenous tradicional medicine: in Vitro anti-giardial activity of plants used in the treatment of diarrhea. Parasitol. Research. PMID: 19153765.

Emmons, L. 1997. Neotropical rainforest mammals. Universidad de Chicago, Chicago, EEUU.

Fowler, J., L. Cohen \& P. Jarvis. 1998. Practical Statistics for Field Biology. Wiley, Nueva York, EEUU.

Fragaszy, D., E. Visalberghi \& L. Redigan. 2004. The complete capuchin. Cambridge University, Cambridge, Inglaterra.

Gilbert, F.F. \& D.G. Doods. 1978. The philosophy and practice of wildlife management. Krieger, Malabar, Florida, EEUU.

Gillespie, T.R. \& C.A. Chapman. 2006. Prediction of parasite infection dynamics in primate metapopulations 
based on attributes of forest fragmentation. Conserv. Biol. 20: 441-448.

Gillespie, T.R. 2006. Noninvasive assessment of gastrointestinal parasite infections in free-ranging primates. Int. J. Primat. 27: 1129-1143.

Gillespie, T.R., C.L. Nunn \& F.H. Leendertz. 2008. Integrative approaches to the study of primate infectious disease: implications for biodiversity conservation and global health. Yearbook Phys. Anthropol. 51: 53-69.

Hausfater, G. \& B.J. Meade. 1982. Alternation of sleeping groves by yellow baboons (Papio cynocephalus) as a strategy for parasite avoidance. Primates 23: 287-297.

Heymann, E.W. 1993. The role of primates in tropical ecosystems, p. 103-108. In W. Barthlott, C.M. Naumann, K. Schmidt-Loske \& K.L. Schuchmann (eds.). Animal-plant Interactions in Tropical Environments. Zoologisches Forschunginstitut/Museo Alexander Koening, Bonn, Alemania.

Ho, A.Y. \& A.S. López. 2002. Outbreak of Cyclospora associated with imported raspberries. Emerg. Inf. Dis. 8: 783-788.

Huffman, M.A. \& C. Chapman. 2009. Primate Parasite Ecology: The Dynamics and Study of Host-Parasite Relationships. Cambridge University, Nueva York, EEUU.

Hugot, J.P., S.L. Gardner \& S. Morand. 1995. The enterobiinae subfam. nov. (Nematoda, Oxyurida) pinworm parasites of primates and rodents. Int. J. Parasitol. 26: $147-159$.

Kageruka, P., J. Brand, H. Taelman \& C. Jonas. 1984. Modified koster staining method for the diagnosis of Cryptosporidium. Am. Soc. Belge Med. Trop. 64: 171-175.

Luna, S., L. Reyes, M. Chinchilla \& G. Catarinella. 2003. Presencia de ooquistes de Cryptosporidium spp. en aguas superficiales en Costa Rica. Parasitol. Latinoam. 57: 63-63.

Martin, P. \& P. Bateson. 1990. Measuring Behaviour. Cambridge University, Nueva York, EEUU.

Murray, P., E. Baron \& M. Pfaller. 1995. Manual of clinical microbiology. ASM, Washington, D.C., EEUU.

Nunn, C.L. \& S. Altizer. 2006. Infectious Diseases in Primates: Behavior, Ecology and Evolution. Oxford Uniersity, Nueva York, EEUU.
Obando V. 2009. Décimo quinto informe estado de La Nación en desarrollo humano sostenible. Informe final. Conservación y biodiversidad. Resultados de la gestión ambiental. (Consultado el 29 Julio 2010, http://www.estadonacion.or.cr/images/stories/informes/015/docs/Armonia/Obando_2009.pdf).

Oppenheimer, J. 1969. Behavior and ecology of the whitefaced monkey, Cebus capucinus, on Barro Colorado Island, C. Z. Tesis de Doctorado, Universidad de Illinois, Urbana-Champaign, EEUU.

Ortega, R. 1997. Isolation of Cryptosporidium parvum and Cyclospora cayetanensis from vegetables collected in markets of an endemic region in Perú. Am. J. Trop. Med. Hyg. 57: 683-686.

Phillips, K.A., M.E. Haas, B.W. Grafton \& M. Yrivarren. 2004. Survey of the gastrointestinal parasites of the primate community at Tambopata National Reserve. J. Zool. 264: 149-51.

Reid, F.A. 1997. A field guide to the mammals of Central America and Southeast Mexico. Oxford University, Nueva York, EEUU.

SINAE - Sistema Nacional de Áreas de Conservación. 2001. Costa Rica. Áreas silvestres protegidas y áreas de conservación. MINAE (Ministerio del Ambiente y Energía), Proyecto ECOMAPAS-INBIO y Gobierno de Noruega. Escala 1:650.000.

Sokal, R.R. and F.J. Rohlf. 1995. Biometry: the principles and practice of statistics in biological research. W.H. Freeman, Nueva York, EEUU.

Stoner, K.E., A.M. González \& S. Maldonado. Infecciones de parásitos intestinales de primates: implicaciones para la conservación. 2005. Universidad y Ciencia 2: 61-72.

Stuart, M., V. Pendergast, S. Rumfelt, S. Pierberg, L. Greenspan, K. Glander \& M. Clarke. 1998. Parasites of wild howlers (Alouatta spp.). Int. J. Primatol. 19: 493-512.

Stuart, M.D. \& K.B. Strier. 1995. Primates and parasites: A case for a multidisciplinary approach. Int. J. Primatol. 16: 577-593.

Terborgh, J. 1992. Diversity and the tropical rain forest. Scientific American Library, Nueva York, EEUU.

Troyo, A., M.E. Solano \& O. Calderón. 2004. Two new species of Listrocarpus Fain (Acari: Atopomelidae) from Cebus capucinus Linnaeus and Saimiri oertedii 
Reinhardt (Primates: Cebidae) in Costa Rica. System. Appl. Acarol., Spec. Public. 18: 1-18.

Urbani, B. 2009. Spatial mapping in wild white-faced capuchin monkeys (Cebus capucinus). Tesis de Doctorado, Universidad de Illinois, Urbana-Champaign, Illinois, EEUU.

Verweij, J.J., J. Vermeer, E.A.T. Brienen, C. Blotkamp, D. laeijendecker, L. Van Lieshout \& A.M. Polderman. 2003. Entamoeba histolytica infections in captive primates. Parasitol. Res. 90: 100-103.

Villalobos, F., A.A. Valerio \& A.P. Retana. 2004. A phylogeny of howler monkeys (Cebidae: Alouatta) base on mitochondrial, chromosomal and morphological data. Rev. Biol. Trop. 52: 665-77.
Visalberghi, E. \& W.C. McGrew. 1997. Cebus meets Pan. Int. J. Primatol. 18: 677-681.

Weber, P., R.T. Brain \& R.L. Owen. 1992. Improved lightmicroscopical detection of microsporidia spores in stool and duodenal aspirates. New Engl. J. Med. 26: 161-166.

Wehncke, E.V., S.P. Hubbell, R.B. Foster \& J.W. Dalling. 2003. Seed dispersal patterns produced by whitefaced monkeys: implications for the dispersal limitation of neotropical tree species. J. Ecol. 91: 677-685.

Zaldivar, M.E., O. Rocha, K.E. Glander, G. Aguilar, A.S. Huertas, R. Sánchez \& G. Wong. 2004. Distribution, ecology, life history, genetic variation and risk of extinction of nonhuman primates from Costa Rica. Rev. Biol. Trop. 52: 679-93. 